\title{
ON AN INEQUALITY OF T. J. WILLMORE
}

\author{
BANG-YEN CHEN
}

ABstract. Willmore proved that the integral of the square of mean curvature $H$ over a closed surface $M^{2}$ in $E^{3}, \int_{M^{2}} H^{2} d V$, is $\geqq 4 \pi$, and equal to $4 \pi$ when and only when $M^{2}$ is a sphere in $E^{3}$. In this paper we give some generalizations of Willmore's result.

Let $x: M^{2} \rightarrow E^{3}$ be an immersion of an oriented closed surface $M^{2}$ into euclidean 3-space $E^{3}$. In [6], [7], Willmore proved the following inequality for the mean curvature $H(p)$ of $M^{2}$ in $E^{3}$ :

$$
\int_{M^{2}} H^{2}(p) d V \geqq 4 \pi,
$$

where the equality holds when and only when $M^{2}$ is imbedded as a sphere. The main aim of this paper is to give some generalizations of the inequality (1).

Acknowledgement. The author would like to express his thanks to Professor T. Nagano for his kind help during the preparation of this paper.

1. Preliminaries. Let $M^{n}$ be an $n$-dimensional oriented closed manifold with an immersion $x: M^{n} \rightarrow E^{n+N}$. Let $F\left(M^{n}\right)$ and $F\left(E^{n+N}\right)$ be the bundles of oriented orthonormal frames of $M^{n}$ and $E^{n+N}$ respectively. Let $B$ be the set of elements $b=\left(p, e_{1}, \cdots, e_{n+N}\right)$ such that $\left(p, e_{1}, \cdots, e_{n}\right) \in F\left(M^{n}\right)$ and $\left(x(p), e_{1}, \cdots, e_{n+N}\right) \in F\left(E^{n+N}\right)$ whose orientation is coherent with the one of $E^{n+N}$, identifying $e_{i}$ with $d x\left(e_{i}\right), i=1, \cdots, n$. Then $B \rightarrow M^{n}$ may be considered as a principal bundle with fibre $S O(n) \times S O(N)$, and $\tilde{x}: B \rightarrow F\left(E^{n+N}\right)$ is naturally defined by $\tilde{x}(b)=\left(x(p), e_{1}, \cdots, e_{n+N}\right)$. Let $B_{v}$ be the set of normal unit vectors of $M^{n}$ in $E^{n+N}$, and $B_{v} \rightarrow M^{n}$ is a sphere bundle whose fibre at $p \in M^{n}$ is $S_{p}^{N-1}$. Let $\tilde{v}: B_{v} \rightarrow S_{0}^{n+N-1}$ be the mapping such that $\tilde{v}(p, e)$ is the unit vector at the origin of $E^{n+N}$ and parallel to $e$.

The structure equations of $E^{n+N}$ are given by

Received by the editors February 10, 1970.

AMS 1969 subject classifications. Primary 5301, 5342; Secondary 5372, 5375.

Key words and phrases. ith mean curvature, Lipschitz-Killing curvature, $\alpha$ th curvatures of first or second kinds, Willmore's inequality, betti number. 


$$
\begin{aligned}
& d x=\sum_{A} \theta_{A} e_{A}, \quad d e_{A}=\sum_{B} \theta_{A B} e_{B}, \\
& \text { (2) } d \theta_{A}=\sum_{B} \theta_{B} \wedge \theta_{B A}, \quad d \theta_{A B}=\sum_{C} \theta_{A C} \wedge \theta_{C B}, \quad \theta_{A B}+\theta_{B A}=0 \text {, } \\
& A, B, C, \cdots=1,2, \cdots, n+N \text {. }
\end{aligned}
$$

where $\theta_{A}, \theta_{A B}$ are differential 1-forms on $F\left(E^{n+N}\right)$. Let $\omega_{A}, \omega_{A B}$ be the induced 1 -forms on $B$ from $\theta_{A}, \theta_{A B}$ by the mapping $\tilde{x}$. Then we have

$$
\begin{aligned}
\omega_{r}=0, \quad \omega_{r i} & =\sum_{j} A_{r i j} \omega_{j}, \quad A_{r i j}=A_{r j i} . \\
& i, j, k, \cdots=1,2, \cdots, n ; r, s, t, \cdots=n+1, \cdots, n+N .
\end{aligned}
$$

From (2) we get

$$
d \omega_{i}=\sum_{j} \omega_{j} \wedge \omega_{j i}, \quad d \omega_{A B}=\sum_{C} \omega_{A C} \wedge \omega_{C B} .
$$

For any $\left(p, e_{r}\right) \in B_{v}$, we put

$$
\mathrm{I}=d x \cdot d x, \quad \mathrm{II}_{r}=d e_{r} \cdot d x .
$$

The eigenvalues $k_{1}\left(p, e_{r}\right), \cdots, k_{n}\left(p, e_{r}\right)$ of $\mathrm{II}_{r}$ relative to I are called the principal curvatures of $M^{n}$ associated with $\left(p, e_{r}\right)$. The $h$ th mean curvature $H_{n}\left(p, e_{r}\right)$ associated with $\left(p, e_{r}\right)$ is defined by the following equation

$$
\operatorname{det}\left(\delta_{i j}+t A_{r i j}\right)=\sum_{h=0}^{n}\left(\begin{array}{l}
n \\
h
\end{array}\right) H_{h}\left(p, e_{r}\right) t^{h},
$$

where $\delta_{i j}$ is the Kronecker delta. If there is no danger of confusion, we shall simply denote $k_{i}\left(p, e_{r}\right)$ and $H_{i}\left(p, e_{r}\right)$ by $k_{i}(p)$ (or $\left.k_{i}\right)$ and $H_{i}(p)$ (or $H_{i}$ ) respectively. It is easy to see that

$$
\left(\begin{array}{l}
n \\
h
\end{array}\right) H_{h}=\sum k_{1} \cdots k_{h}, \quad h=1,2, \cdots, n,
$$

and $H_{0}=1$. Throughout this paper, we simply denote $H_{n}\left(p, e_{r}\right)$ by $K\left(p, e_{r}\right) . K\left(p, e_{r}\right)$ is called the Lipschitz-Killing curvature at $\left(p, e_{r}\right)$.

2. $\alpha$ th curvature of first and second kinds. Let $M^{2}$ be a surface immersed in $E^{2+N}$. Let $\left(p, e_{1}, e_{2}, \bar{e}_{3}, \cdots, \bar{e}_{2+N}\right)$ be a local cross section of $B \rightarrow F\left(M^{2}\right)$ and for any $e$ in $S_{p}^{N-1}, p \in U$, put $e=e_{2+N}=\sum_{r} \xi_{r} \bar{e}_{r}(p)$. Denoting the restriction of $A_{r i j}$ on the image of this local cross section by $\bar{A}_{r i j}$, we may put

$$
A_{2+N i j}=\sum_{r} \xi_{r} \bar{A}_{r i j}
$$


From (3) and (6), we get

$$
\begin{aligned}
K(p, e) & =\operatorname{det}\left(\sum_{r} \xi_{r} \bar{A}_{r i j}\right) \\
& =\left(\sum_{r} \xi_{r} \bar{A}_{r 11}\right)\left(\sum_{s} \xi_{s} \bar{A}_{s 22}\right)-\left(\sum_{t} \xi_{t} \bar{A}_{t 12}\right)^{2} .
\end{aligned}
$$

The right-hand side is a quadratic form of $\xi_{3}, \cdots, \xi_{2+N}$. Hence, by choosing a suitable cross section, we can write $K(p, e)$ as

$$
K(p, e)=\sum_{r} \lambda_{r-2} \xi_{r} \xi_{r}, \quad \lambda_{1} \geqq \lambda_{2} \geqq \cdots \geqq \lambda_{N} .
$$

We call this local cross section of $B \rightarrow F\left(M^{2}\right)$, the Frenet cross section in the sense of Ōtsuki, and the frame $\left(p, e_{1}, e_{2}, \bar{e}_{3}, \cdots, \bar{e}_{2+N}\right)$ the Otsuki's frame [5]. We call the curvature $\lambda_{\alpha}$, the $\alpha$ th curvature of the second kind. With respect to this Ōtsuki's frame the curvatures:

$$
\mu_{\alpha}(p)=H_{1}\left(p, \bar{e}_{\alpha+2}\right)
$$

are called the $\alpha$ th curvature of the first kind. By means of the method of definitions, $\mu_{\alpha}, \lambda_{\alpha}$ are defined continuous on the whole manifold $M^{2}$ and are differentiable on the open subset in which $\lambda_{1}>\lambda_{2}$ $>>\lambda_{N}$.

With respect to the Ōtsuki frame, we have [5]

$$
\begin{aligned}
\omega_{1 r} \wedge \omega_{2 r}=\lambda_{r-2} d V, \quad d V & =\omega_{1} \wedge \omega_{2}, r=3, \cdots, 2+N, \\
G(p) & =\sum_{\alpha=1}^{N} \lambda_{\alpha}(p),
\end{aligned}
$$

where $G(p)$ denotes the Gaussian curvature of $M^{2}$ in $E^{2+N}$, and we also have

$$
H_{1}(p, e)=\sum_{\alpha=1}^{N} \cos \theta_{\alpha} \mu_{\alpha}(p), \quad e=\sum_{r} \cos \theta_{r-2} \bar{e}_{r} .
$$

As in [2], [3], [5], we know that the forms:

$$
d \sigma=\omega_{2+N, 3} \wedge \cdots \wedge \omega_{2+N, 1+N}, \text { and } d V \wedge d \sigma
$$

can be regarded as the volume elements of $S_{p}^{N-1}$ and $B_{v}$ respectively.

\section{Some generalizations of Willmore's inequality.}

TheOREM 1. Let $x: M^{2} \rightarrow E^{2+N}$ be an immersion of an oriented closed surface $M^{2}$ into $E^{2+N}$. Then the sum of the squares of the ath curvatures 
of the first kind satisfies the following inequality:

$$
\int_{M^{2}}\left(\sum_{\alpha=1}^{N} \lambda_{\alpha}^{2}\right) d V \geqq 4 \pi,
$$

where the equality holds when and only when $M^{2}$ is imbedded as a sphere in a 3-dimensional linear subspace of $E^{2+N}$.

Proof. Let $\left(p, e_{1}, e_{2}, \bar{e}_{3}, \cdots, \bar{e}_{2+N}\right)$ be an Ōtsuki's frame, then by (9) we know that the Lipschitz-Killing curvature $K(p, e)$ satisfies

$$
\begin{aligned}
\int_{B_{v}} K(p, e) d V \wedge d \sigma & =\int_{B_{v}}\left(\lambda_{1}(p) \cos ^{2} \theta_{1}+\cdots+\lambda_{N}(p) \cos ^{2} \theta_{N}\right) d V \wedge d \sigma \\
& =\frac{c_{N+1}}{2 \pi} \int_{M^{2}}\left(\sum_{\alpha=1}^{N} \lambda_{\alpha}^{2}(p)\right) d V=\frac{c_{N+1}}{2 \pi} \int_{M^{2}} G(p) d V .
\end{aligned}
$$

Thus by the Gauss-Bonnet formula, we have

$$
\int_{B_{v}} K(p, e) d V \wedge d \sigma=(2-2 g) c_{N+1}
$$

where $g$ denotes the genus of $M^{2}$ and $c_{N+1}$ denotes the volume of the unit $(N+1)$-sphere. On the other hand, by an inequality of ChernLashof [3], we have

$$
\int_{B_{\text {v }}}|K(p, e)| d V \wedge d \sigma \geqq(2+2 g) c_{N+1} .
$$

Therefore, if we set

$$
V+=\left\{(p, e) \in B_{v}: K(p, e) \geqq 0\right\}, \quad V-=\left\{(p, e) \in B_{v}: K(p, e)<0\right\},
$$

then, by (16) and (17), we get

$$
\int_{V+} K(p, e) d V \wedge d \sigma \geqq 2 c_{N+1} .
$$

It is easy to see that the equality of (19) holds when and only when

$$
\int_{B_{v}}|K(p, e)| d V \wedge d \sigma=(2+2 g) c_{N+1}
$$

Now, we have the following identity:

$$
\left(k_{1}+k_{2}\right)^{2}=\left(k_{1}-k_{2}\right)^{2}+4 k_{1} k_{2} \text {. }
$$

Hence we get 


$$
\begin{aligned}
\int_{B_{v}}\left(H_{1}(p, e)^{2}\right) d V \wedge d \sigma & \geqq \int_{\nabla_{+}}\left(H_{1}(p, e)^{2}\right) d V \wedge d \sigma \\
& \geqq \int_{V+} K(p, e) d V \wedge d \sigma
\end{aligned}
$$

Therefore, by (19), we get

$$
\int_{B_{v}}\left(H_{1}(p, e)\right)^{2} d V \wedge d \sigma \geqq 2 c_{N+1} .
$$

On the other hand, by (14) and the following formulas:

$$
\begin{aligned}
\int_{S_{p}^{N-1}} \cos \theta_{\alpha} \cos \theta_{\beta} d \sigma & =c_{N+1} / 2, & & \text { if } \alpha=\beta, \\
& =0, & & \text { if } \alpha \neq \beta,
\end{aligned}
$$

we have

$$
\begin{aligned}
\int_{B_{v}}\left(H_{1}(p, e)^{2}\right) d V \wedge d \sigma & =\sum_{\alpha, \beta=1}^{N} \int_{B_{v}} \mu_{\alpha}(p) \mu_{\beta}(p) \cos \theta_{\alpha} \cos \theta_{\beta} d V \wedge d \sigma \\
& =\frac{c_{N+1}}{2 \pi} \int_{M^{2}}\left(\sum_{\alpha} \mu_{\alpha}^{2}(p)\right) d V .
\end{aligned}
$$

Hence, by (22) and (23), we get (15). Now, suppose that the equality of (15) holds, then, by (19), (20) and (21), we get

$$
H_{1}(p, e)^{2}=K(p, e), \quad \text { and } \quad k_{1}(p, e)=k_{2}(p, e),
$$

for all $(p, e)$ in $B_{v}$. Thus, by (9) and (24), we know that the last curvature of the second kind $\lambda_{N} \geqq 0$, for all $p$ in $M^{2}$. Hence, by Lemma 1 of [2], we know that $M^{2}$ is imbedded as a convex surface in a 3dimensional linear subspace, say $E^{3}$, of $E^{2+N}$. Thus, by (24), we know that $M^{2}$ is imbedded as a sphere in $E^{3}$. Conversely, if $M^{2}$ is imbedded as a sphere in a 3-dimensional linear subspace of $E^{2+N}$, then it is easy to see that the equality of (15) holds. This completes the proof of the theorem.

In the following, we assume that $x: M^{2 m} \rightarrow E^{2 m+1}$ is an immersion of an oriented even-dimensional closed manifold $M^{2 m}$ into $E^{2 m+1}$. Let $\bar{e}$ denote the outer normal vector on $M^{2 m}$ in $E^{2 m+1}$. Set

$$
g(p)=H_{m}(p)^{2}-K(p),
$$

where $H_{m}(p)=H_{m}(p, \bar{e})$ and, $K(p)=K(p, \bar{e})$. By Theorem 1 of [1], we have the following proposition. We omit the proof. 
Proposition 2. Let $x: M^{2 m} \rightarrow E^{2 m+1}$ be an immersion of an oriented $2 m$-dimensional closed manifold $M^{2 m}$ in $E^{2 m+1}$ with the following property:

$$
\left\{p \in M^{2 m}: g(p) \geqq 0\right\} \supseteq\left\{p \in M^{2 m}: K(p) \geqq 0\right\} .
$$

Then we have the following inequality:

$$
\int_{M^{2 m}} H_{m}(p)^{2} d V \geqq \frac{1}{2} c_{2 m} \sum_{i} \beta_{2 i}
$$

where $\beta_{i}$ denotes the ith betti number of $M^{2 m}$. The equality of (26) holds when and only when the immersion $x$ is a minimal imbedding [1].

REMARK. If $m=1$, then (A) always holds.

THEOREM 3. Let $x: M^{2 m} \rightarrow E^{2 m+1}$ be an immersion of an oriented $2 m$ dimensional closed manifold $M^{2 m}$ in $E^{2 m+1}$ with nonnegative principal curvatures. Then the mth mean curvature $H_{m}$ satisfies the following:

$$
\int_{M^{2 m}}\left(H_{m}(p)^{2}\right) d V \geqq \frac{1}{2} c_{2 m} \sum_{i} \beta_{i},
$$

where the equality holds when and only when $M^{2 m}$ is imbedded as a sphere.

Proof. By the assumption, $k_{1}, \cdots, k_{n} \geqq 0$, we have $[4$, pp. 104 105]

$$
H_{m}^{2} \geqq K \geqq 0,
$$

where the equality holds when and only when $k_{1}=k_{2}=\cdots=k_{n}$. Thus, by Proposition 2, we have (26). Furthermore, by Theorem 3 of [1], we know that all odd-dimensional betti numbers of $M^{2 m}$ vanish. Hence, by (26), we get (27). Now, suppose that the equality of (27) holds. Then we get $H_{m}^{2}(p)=K(p)$ for all $p \in M^{2 m}$. Hence, we get $k_{1}(p)=\cdots=k_{n}(p)$ for all $p \in M^{2 m}$. Thus $M^{2 m}$ is imbedded as a sphere in $E^{2 m+1}$. This completes the proof of the theorem.

\section{REFERENCES}

1. B-y. Chen, Some integral formulas of the Gauss-Kronecker curvature, Kodai Math. Sem. Rep. 20 (1968), 410-413. MR 38 \#2796.

2. - Surfaces of curvature $\lambda_{N}=0$ in $E^{2+N}$, Kōdai Math. Sem. Rep. 20(1969), 331-334.

3. S. S. Chern and R. K. Lashof, On the total curvature of immersed manifolds. II, Michigan Math. J. 5 (1958), 5-12. MR 20 \#301. 
4. G. H. Hardy, J. E. Littlewood and G. P6lya, Inequalities, Cambridge Univ. Press, New York, 1934.

5. T. Ōtsuki, On the total curvature of surfaces in Euclidean spaces, Japan. J. Math. 35 (1966), 61-71. MR 34 \#692.

6. T. J. Willmore, Note on embedded surfaces, An. Ști. Univ. "Al. I. Cuza" Iaşi. Secț. Ia Mat. 11B (1965), 493-496. MR 34 \#1940.

7. - Mean curvature of immersed surface, An Şti. Univ. "Al. I. Cuza" Iaşi. Secț. Ia Mat. 14 (1968), 99-103. MR 38 \#6496.

University of Notre Dame, Notre Dame, Indiana 46556

Michigan State University, East Lansing, Michigan 48823 